\title{
ANÁLISIS GEOAMBIENTAL DEL ÁREA DE PASTOREO DE LA UNIDAD BÁSICA DE PRODUCCIÓN COOPERATIVA ANTONIO MACEO, MOA, CUBA.
}

\author{
Y. BORGES-TERRERO ${ }^{1}$, Y. ALMAGUER-CARMENATE ${ }^{2}$, S. GARCÍA-CRUZ ${ }^{3}$, A.M. OCA-RISCO ${ }^{4}$ \\ Universidad de $\mathrm{Moa}^{1,3,4}$ \\ Universidad Autónoma de Coahuila ${ }^{2}$ \\ yborgest@ismm.edu.cu ${ }^{1}$
}

Artigo submetido em 18/06/2019 e aceito em 16/12/2019

DOI: $10.15628 /$ holos.2019.8735

\section{RESUMEN}

Se evalúan los procesos geodinámicos externos presentes en el área de pastoreo de la Unidad Básica de Producción Cooperativista (UBPC) Antonio Maceo, Moa, Holguín, Cuba. Con el fin de establecer la incidencia de dichos procesos en las producciones de leche. La metodología de la investigación consta de tres etapas de trabajo: una fase preliminar, donde se accedió a toda la información bibliográfica existente sobre el tema; una segunda fase de trabajos de campo, que incluye el reconocimiento del área de pastoreo y la caracterización de los fenómenos geodinámicos exógenos presentes y por último la fase de gabinete, en la cual se interpretan los resultados. Se obtiene que el área de pastoreo es afectada principalmente por los procesos de meteorización química y erosión por cárcavas, lo cual provoca la remoción de las capas superficiales de los suelos y poca densidad de vegetación herbácea, lo que influye en la disponibilidad de nutrientes necesarios en el área de pastoreo, por lo tanto se evidencia en la baja producción de leche.

\section{EVALUATION OF ENVIRONMENTAL IMPACT OF THE CONSTRUCTION OF PROTECTING BUILDING WORK IN MOA, HOLGUÍN}

\begin{abstract}
The external geodynamic processes present in the grazing area of the Basic Unit of Cooperative Production (UBPC) Antonio Maceo, Moa, Cuba, are evaluated. In order to establish the incidence of these processes in milk production. The methodology of the investigation consists of three stages of work: a preliminary phase, where all the existing bibliographic information on the subject was accessed; a second phase of fieldwork, which includes the recognition of the grazing area and
\end{abstract} phenomena present and finally the cabinet phase, in which the results are interpreted. It is obtained that mainly the processes of chemical weathering and erosion affect the grazing area by gullies, which causes the removal of the superficial layers of the soils and low density of herbaceous vegetation, which influences the availability of necessary nutrients in the grazing area; therefore, it is evident in the low milk production. 


\section{INTRODUCCIÓN}

El medio ambiente está constituido por: "un sistema de elementos abióticos, bióticos y socioeconómicos con que interactúa el hombre, a la vez que se adapta al mismo, lo transforma y lo utiliza para satisfacer sus necesidades" (Chaviano, 2016), este enfoque, considera el medio físico, el biológico y el socio-económico. Esto denota que, en el marco de los análisis ambientales, la geología tiene como objeto de estudio el medio físico, en especial los procesos que se desarrollan en la litosfera y la hidrosfera.

Yakabi (2016) expone el siguiente concepto; suelo es la parte superficial de la litosfera. Constituida por una mezcla variable de partículas minerales, materia orgánica, aire y agua. Se forma a través de un conjunto de procesos físicos, químicos y biológicos sobre el medio rocoso original (meteorización), siendo el soporte material para el desarrollo de organismos vivos.

Muñiz (2015) en su artículo resume los principales logros y desafíos de la ciencia del suelo en Cuba en los 50 años transcurridos desde la fundación del Instituto de Suelos, período en que tales estudios se acometieron de forma sistemática y metódica en el país. Se presentan los principales resultados; entre ellos se mencionan: el desarrollo e introducción de la clasificación genética de los suelos de Cuba, en sus diferentes versiones; la clasificación agroproductiva de los suelos de Cuba; el mapa a escala 1: 25000 de los suelos de Cuba y el establecimiento de las bases del Servicio de Suelos que brindan a los agricultores, tanto el Instituto de Suelos como el Instituto de Investigaciones de la Caña de Azúcar.

Lo antes planteado lo presupone Fernández (2017), el cual explica que el medio físico es el objeto de la geología y se entiende como "el conjunto de componentes predominantemente abióticos, como los materiales terrestres (suelo, rocas, agua, aire) y tipos de energía incluyendo sus modificaciones resultantes de la acción biológica y humana".

Por más de 50 años el municipio de Moa ha mantenido un desarrollo minero-metalúrgico considerable, lo que ha conducido a una serie de factores que conduce a la pérdida de la calidad de los suelos. Por la naturaleza del medio geológico y las grandes reservas ferroniquelíferas que posee, es necesario desarrollar estudios que permitan aplicar medidas para lograr obtener mayores producciones.

Por ello surge la presente investigación "Análisis geoambiental del área de pastoreo de la Unidad Básica de Producción Cooperativa Antonio Maceo, Moa, Cuba" a partir del siguiente problema: Insuficiente conocimiento del área dedicada al pastoreo en la UBPC Antonio Maceo de Moa.

Con vista a la solución de este problema se propone como objetivo general de la investigación, evaluar los procesos geodinámicos de superficie presentes en el área de pastoreo de la UBPC Antonio Maceo. 


\section{REVISIÓN BIBLIOGRÁFICA}

\section{Conceptos y definiciones}

Desde las postrimerías del pasado siglo XX y a las puertas del presente siglo XXI la lucha por la conservación del Medio Ambiente y el desarrollo sostenible ha pasado a ser una de las principales prioridades políticas y estatales en todos los países del orbe, en consonancia con el gradual agravamiento de la situación ambiental mundial ( García, 2018).

Erosión o degradación de los suelos. Es la pérdida del mismo, principalmente por factores como las corrientes de agua y de aire, en particular en terrenos secos y sin vegetación, además el hielo y otros factores. La erosión del suelo reduce su fertilidad porque provoca la pérdida de minerales y materia orgánica (Rodríguez, 2015).

\section{Calificación de los procesos erosivos (CCD/PNUMA, 1995)}

- Impacto de las gotas de lluvia sobre el suelo: dispersión de los agregados del suelo en sus partículas elementales (texturales). Puede formarse una costra superficial o un sello (sellado) que impide una adecuada

- Erosión en Cárcavas: suelo arrastrado por el agua infiltración del agua generando su pérdida por escorrentía superficial.

- Erosión laminar: pérdida de suelo generada por circulación superficial difusa del agua de escorrentía.

- Erosión en Surcos: suelo arrastrado por el flujo del agua que se canaliza y jerarquiza generando surcos. Que al generar cárcavas (estas suelen comenzar en forma de surcos).

- Erosión en "Badlands": erosión en cárcavas profundas generalizada, que llega a eliminar toda la capa de suelo dando lugar a un paisaje "abarrancado".

- Erosión por sufusión (piping): desarrollo de una red de drenaje sub-superficial que termina por colapsarse. Suele acompañar a los paisajes de "bandalnds).

- Bio-erosión: erosión de las capas subsuperficiales del suelo causada por la acción de organismos vivos.

- Erosión Mecánica: pérdida de suelo causada por las labores de la labranza.

- Nivelamiento del terreno: pérdida de suelo debida a la modificación humana del perfil original de una ladera o la construcción de terrazas.

- Erosión de los cauces fluviales: génesis de paisajes fluviales por incisión de las aguas pluviales o por el desplazamiento lateral de los propios cursos (erosión de márgenes fluviales).

- Erosión costera o litoral: erosión costera debida al efecto del oleaje y las mareas, por la que el mar gana terreno en detrimento de las superficies emergidas.

- Erosión glaciar: génesis de los paisajes frías, glaciares y periglaciares a causa de los flujos de hielo. Su avance suele acarrear la pérdida total de los suelos.

- Deslizamientos de masa_someros: desplazamiento de suelo y a veces regolito que deja una cicatriz en hondonada y un lóbulo frontal sobresaliente. A menudo, muchos deslizamientos someros evolucionan hacia flujos de clastos (piedras, cantos bloques de rocas). En principio, si no actúan otros procesos erosivos se puede hablar más de desplazamiento que de pérdida del recurso. 
- Erosión eólica o deflación: pérdida del suelo debido al efecto erosivo del viento el consiguiente arrastre de los materiales edáficos arrancados.

- Erosión eólica o corrosión: desprendimiento de partículas (abrasión) debido al impacto de partículas previas suspendidas o arrastradas por el viento que genera modelados 0 esculpidos muy característicos y a veces bellos que reciben diversas denominaciones.

Meteorización. La meteorización es la desintegración y descomposición de una roca en la superficie terrestre o próximo a ella como consecuencia de su exposición a los agentes atmosféricos, con la participación de agentes biológicos. La meteorización involucra un conjunto de reacciones químicas en las que los productos sirven de reactivos para síntesis subsiguientes. $\mathrm{Si}$ el proceso de la meteorización ocurre en la superficie del suelo se llama meteorización edafoquímica y si ocurre en capas más profundas como el horizonte C o más se llama meteorización geoquímica (Rodríguez, 2015).

Sedimentación. Es el proceso por el cual los materiales son transportados por distintos agentes (escorrentía, glaciares, viento) y procedentes de la erosión y la meteorización de las rocas son depositados, pasando a ser sedimentos. El tipo más extendido de sedimentación ocurre cuando los derrubios (restos sólidos arrancados a las rocas) transportados por una corriente de agua, se depositan en el fondo del cauce de un río, en una llanura de inundación, en un embalse, en un canal artificial, o en un dispositivo artificial construido especialmente para separar la materia en suspensión. Toda corriente de agua, caracterizada por su caudal, tirante de agua, velocidad y forma de la sección tiene una capacidad de transportar material sólido en suspensión (además de moléculas en disolución). El cambio de alguna de estas características de la corriente puede hacer que el material transportado se deposite o precipite; o que el material existente en el fondo o los márgenes del cauce sea erosionado (Rodríguez, 2015).

\section{Investigaciones precedentes}

Carreño et al. (2005), presenta una síntesis formalizada de los diferentes enfoques de los métodos empleados para evaluar la erosión, los datos que requieren y el tipo de resultado que brindan, con el objetivo de ponerlas a disposición de los especialistas dedicados a esta temática de investigación teniendo en cuenta que hasta el presente, en Cuba está muy limitado el empleo de algunas de las metodologías, que tienen amplio uso a nivel mundial. En Cuba el método geográfico - comparativo, ha sido el más empleado en los estudios de erosión utilizando en calidad de patrones, perfiles típicos de cada agrupamiento. Sólo en años recientes se han incorporado a las investigaciones los modelos de erosión, fundamentalmente la USLE y la cartografía temática de factores. La evaluación de la erosividad de la lluvia en general se realiza sin considerar la energía cinética o algunos de los índices de agresividad climática ampliamente reconocidos en la literatura mundial especializada.

Martínez (2014), mediante una técnica de teledetección detecta cambios en la cubierta vegetal tanto esporádicos como continuos a partir del seguimiento multitemporal de imágenes a escala regional usando el NOAA- AVHRR. Esta metodología se utilizó fundamentalmente en zonas áridas y semiáridas, consideradas como zonas muy sensibles a unos de los procesos de degradación más destructivos del suelo y la vegetación, el proceso de desertificación. 
Fernández (2017), realiza un estudio sobre los factores que influyen en la desertificación en el sector Cerro Miraflores- Playa La Vaca, municipio Moa. La aplicación de métodos de cartografía geológica y descripción de afloramientos, orientada a la determinación de procesos geológicos y las prácticas de la actividad humana que se ejerce sobre el medio; el procesamiento de la información se realiza con métodos de cartografía digital. Como resultados se describen los factores que contribuyen al proceso de desertificación; el uso del suelo en algunas áreas ha eliminado la vegetación natural y transformado el medio, disminuyendo la fertilidad de los suelos y dañando cursos fluviales; en zonas de altas las pendientes los procesos erosivos son más intensos y la densidad de vegetación disminuye en los sectores de mayor altitud, pendientes y donde han sido afectados por incendios forestales.

García (2018) realiza un análisis de los factores que inciden en la degradación de los suelos mediante métodos de cartografía digital, con vista a realizar futuros análisis de vulnerabilidad y riesgos ante este fenómeno y la pérdida de biodiversidad. Se caracteriza desde el punto de vista geológico la cuenca y se analizan los factores naturales y antrópicos que influyen sobre la degradación de los suelos. Como resultados se describen los factores que contribuyen al proceso de degradación: El uso del suelo en algunas áreas ha eliminado la vegetación natural y transformada el medio, disminuyendo la fertilidad de los suelos y dañando cursos fluviales. En zonas de altas las pendientes $\left(15^{\circ}-60^{\circ}\right)$ y de mayor altitud los procesos erosivos son más intensos por lo que la densidad de vegetación disminuye. En las zonas de baja densidad de vegetación la acción antrópica es la principal causante de perdida de suelo. Los escenarios prospectivos de degradación indican el deterioro de la productividad biológica de los suelos y pérdida de biodiversidad ocuparán el 58,71\% del área total de la cuenca.

\section{MATERIAL Y MÉTODOS}

\subsection{Caracterización del área de estudio}

\subsubsection{Ubicación del área de estudio}

La UBPC Antonio Maceo se encuentra localizada en el sector La Granja, perteneciente al poblado Centeno, municipio Moa, provincia Holguín. Limita al norte con la carretera central Moa Sagua de Tánamo, al sur con la mina de la fábrica niquelífera Pedro Sotto Alba y el río Cabaña, al este con la empresa Pedro Sotto Alba, el poblado de La Melba y el Reparto Armando Mestre y al oeste con el Cerro Miraflores (figura 1). 


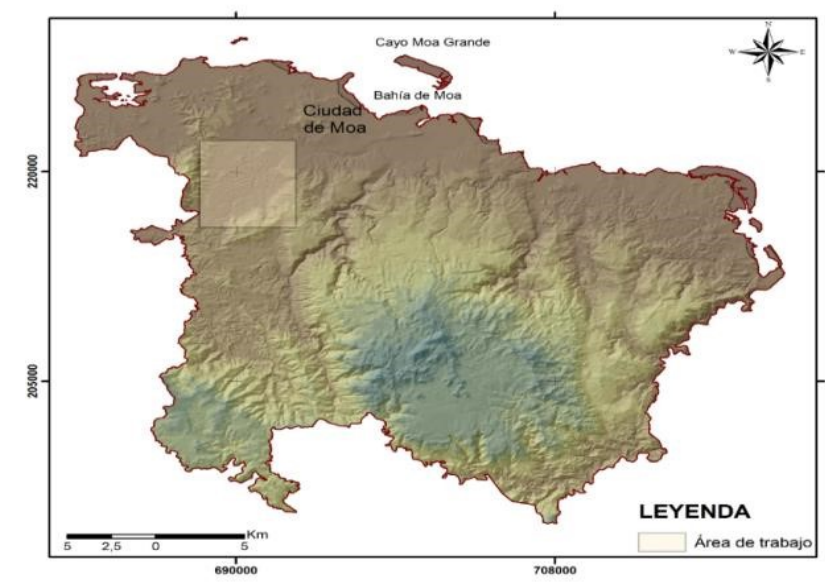

Figura 1: Ubicación del Sector la Granja (elaboración propia)

\subsubsection{Clima}

Presenta un clima tropical con abundantes precipitaciones, al ser una de las áreas de mayor pluviometría del país. Se encuentra estrechamente relacionada con el relieve montañoso que se desarrolla en la región y con la dirección de los vientos alisios provenientes del Océano Atlántico cargado de humedad (figura 2).

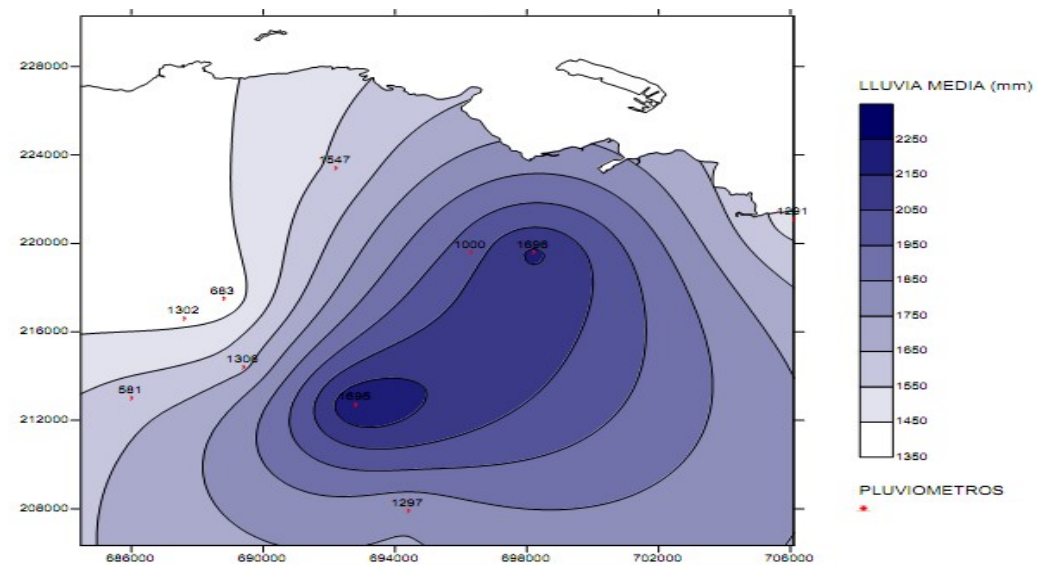

Figura 2: Mapa pluviométrico del territorio de Moa. Escala 1:50 000 (Instituto Nacional de Recursos Hidráulicos, Habana, 2005) (tomado de Ramírez, 2015)

\subsubsection{Relieve}

Se caracteriza por una alta complejidad, con predominio del sistema montañoso hacia la parte este, cota máxima de 1139 m sobre el nivel del mar y ondulado hacia el norte, zona correspondiente a la región costera. En el área aparecen varias formas del relieve, teniendo mayor relevancia la zona de llanuras, en especial las llanuras fluviales erosivas-acumulativas: se localizan principalmente desde el centro del valle de la cuenca hasta el norte de la misma; la superficie topográfica pierde su regularidad al aparecer sectores de hasta 9o de pendiente, se condiciona la existencia de procesos erosivos. Los sedimentos que se acumulan en estas llanuras son de origen fluvial y su deposición es generalmente de carácter temporal, siendo removidos con frecuencia en los períodos de crecida (Batista, 1998). 
La formación de estas llanuras está relacionada con la acción conjunta de diferentes procesos morfogénicos que en ella han actuado, influenciado por los procesos fluviales y marinos. Al sur del área de estudio predomina el relieve montañoso, ocupado por la mayor extensión las montañas bajas aplanadas y las premontañas aplanadas ligeramente diseccionadas (figura 3).

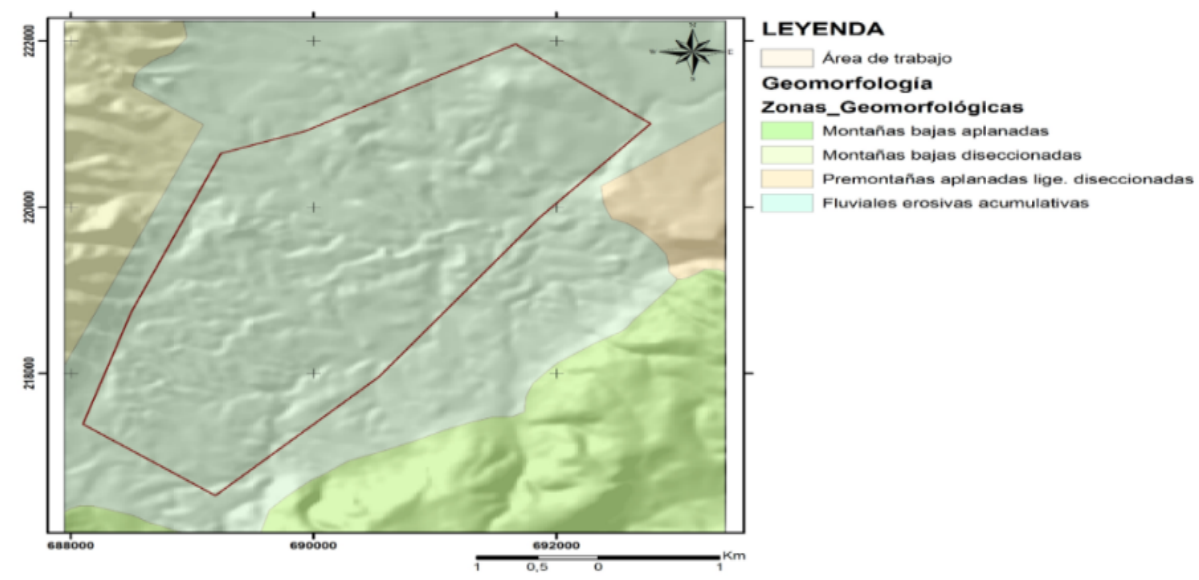

Figura 3: Mapa geomorfológico del área de estudio (modificado de Rodríguez, 1999)

\subsubsection{Caracterización geológica}

Representada por una variada composición litológica. Estratigráficamente está compuesta por las rocas del Complejo Ofiolítico (peridotitas, gabros, basaltos) y los sedimentos fluviales del Cuaternario. Según el mapa geológico (figura 4) el área se sitúa casi exclusivamente sobre cuerpos de gabros que tienen una estructura de grandes bloques y serpentinitas muy meteorizadas.

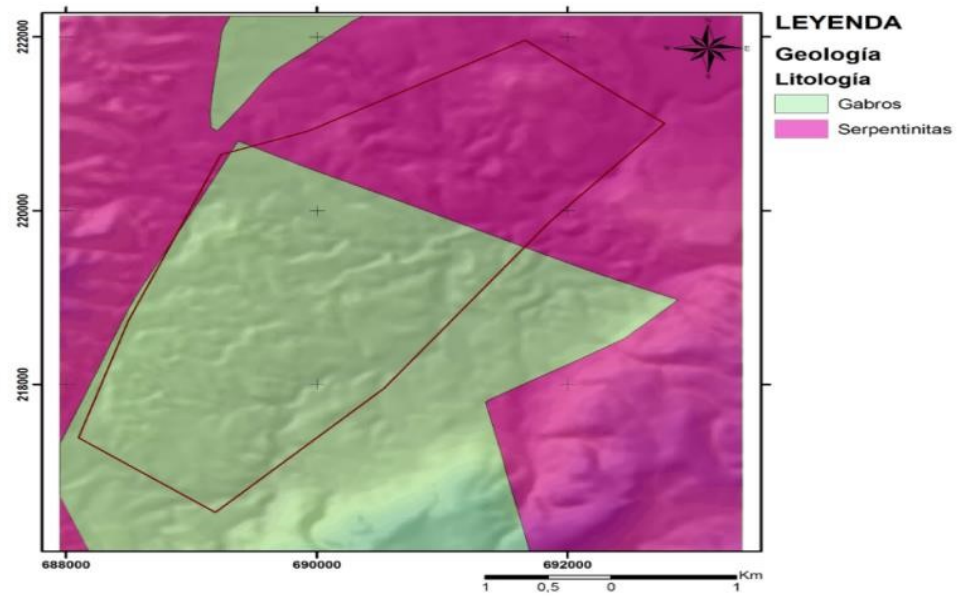

Figura 4: Mapa geológico del área de estudio (modificado de Rodríguez, 1999)

\subsubsection{Caracterización hidrogeológica}

El área de estudio pertenece al Complejo acuífero de las lateritas: se extiende por casi toda la zona ocupando gran parte del área. Litológicamente está formado por potentes cortezas de intemperismo. Representa un acuitardo, debido al predominio de aguas capilares y de potencias considerables de lateritas, que alcanzan valores de $30 \mathrm{~m}$, con un marcado desarrollo de los procesos de capilaridad, donde los ascensos capilares de las aguas pueden alcanzar más de $20 \mathrm{~m}$. 
La fuente de alimentación de estas aguas son las precipitaciones atmosféricas. Por su composición química son aguas hidrocarbonadas-magnésicas y sódicas de baja mineralización.

La metodología utilizada tiene por objetivo mostrar una forma de evaluación a seguir, que comprenda la descripción de los procesos geodinámicos de superficie en el área de pastoreo. Se desarrolla en tres etapas de trabajo, las cuales se describen a continuación:

I Etapa. Preliminar. Recopilación y revisión de la información existente

En esta etapa se realiza una búsqueda de información bibliográfica en: una serie de artículos científicos, trabajos investigativos, revistas, tesis de grado, de maestría y doctorales, así como informes relacionados con el tema a tratar. Se utiliza documentación del Centro de Información del ISMM, de sitios web de internet especializados en el tema y entrevistas a los pobladores y trabajadores de La Granja.

II Etapa. Trabajo de Campo

Los trabajos de campo se realizan con el objetivo de describir en el terreno los procesos geológicos, identificar los tipos de rocas, suelo y los impactos fundamentales de la actividad antrópica sobre el medio. Se revisan las fotos aéreas descargadas del programa Google Earth y a la interpretación de la documentación realizada en 14 puntos del área de pastoreo a una distancia de $250 \mathrm{~m}$, se utilizan fichas normalizadas con el objetivo de homogenizar los datos y facilitar el procesamiento de la información, los elementos tenidos en cuenta son:

Realización de marchas de reconocimiento para la descripción geológica de afloramientos e identificación de procesos geodinámicos que se desarrollan en el sector.

Descripción de los tipos de suelos e identificación de criterios de productividad biológica, en base a la densidad de vegetación y desarrollo del follaje.

Identificación de impactos antrópicos sobre los factores ambientales en el área.

Se utilizó como equipamiento: Mapas topográficos, GPS, Brújula, Mapa geológico, Libreta, lápiz, marcador, Piqueta, Pico, Cámara fotográfica.

III Etapa. Procesamiento de la información

En esta etapa al obtener las informaciones necesarias de campo y laboratorio que garantizan el cumplimiento de los objetivos de la investigación se evalúan y procesan mediante herramientas de Office 2010, entre ellas Microsoft Excel y Word, etc. Para luego ser interpretados mediante tablas y gráficos que forman parte de la memoria escrita.

\section{RESULTADOS Y DISCUSIÓN}

Las unidades ganaderas, requieren de un proceso de años de trabajo e inversión de capital, basados en el mejoramiento y buen manejo de las praderas, del conocimiento de las técnicas modernas de producción y la correcta toma de decisiones, sustentadas en registros de producción e información de mercado. Todo esto, determina que los sistemas de producción, no tengan un patrón único y definido de cómo producir más, sino que se deben considerar 
una combinación de factores que están disponibles en el medio interno y externo de la unidad productiva. Razones por las cuales se realiza la descripción de los procesos geodinámicos externos en el área de pastoreo (figura 5) que pudiera influir en la baja productividad.

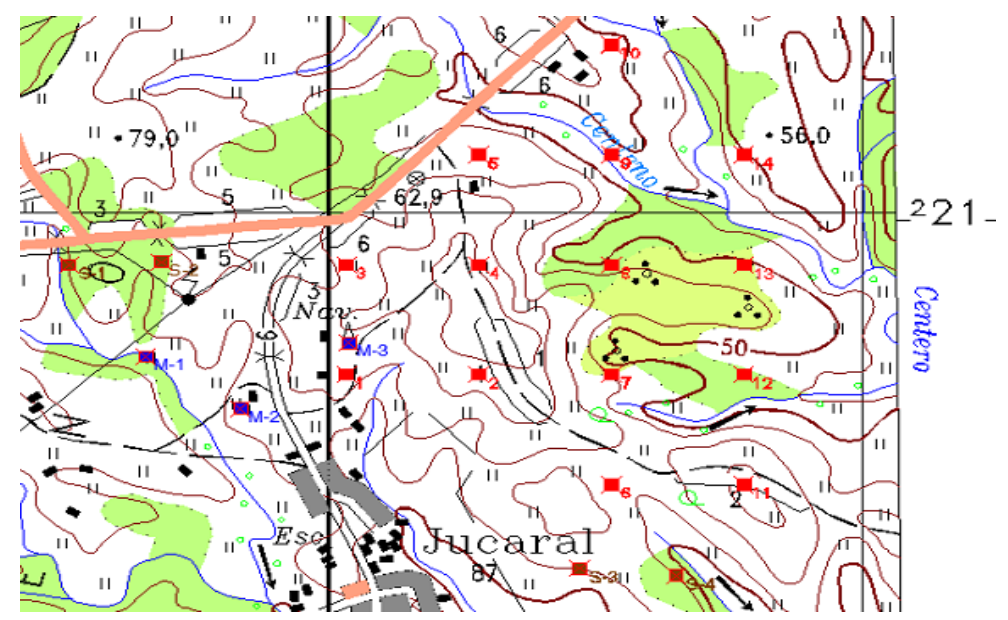

Figura 5: Ubicación de los puntos de reconocimiento geológico dentro del área de estudio

A continuación se describen los 14 puntos de reconocimiento geológico:

Punto 1

Ubicación: Este punto se encuentra a $50 \mathrm{~m}$ al este del camino que da entrada al poblado La Granja.

Coordenadas: X: $690033.9 \quad$ Y: 220635.7

Litologías: Se observa corteza de meteorización (figura 6) de color pardo-rojizo con tonalidad amarillenta, de tipo ultramáfica, con predominio de fragmentos de roca serpentinita.

Vegetación. La vegetación es poco densa y predominan los charracales. Suelos. Desde el punto de vista edafológico los suelos son ferruginosos se observa un color pardo- rojizo, con presencia de minerales oxidados:

$60 \%$ minerales oxidados, $30 \%$ arcilla, $10 \%$ cromita estable y otros minerales

En el corte es insitu, con roca alterada y horizonte prácticamente incipiente, no hay indicios de redeposición, sin presencia de estratificaciones y sin perdigones.

Red fluvial: No hay presencia de arroyos.

Fenómenos geodinámicos: Se observan cárcavas de pequeñas dimensiones producto al escurrimiento pluvial y no afloran las rocas.

Acción antrópica: Presencia de la vaquería y de los asentamientos poblacionales en la comunidad. 


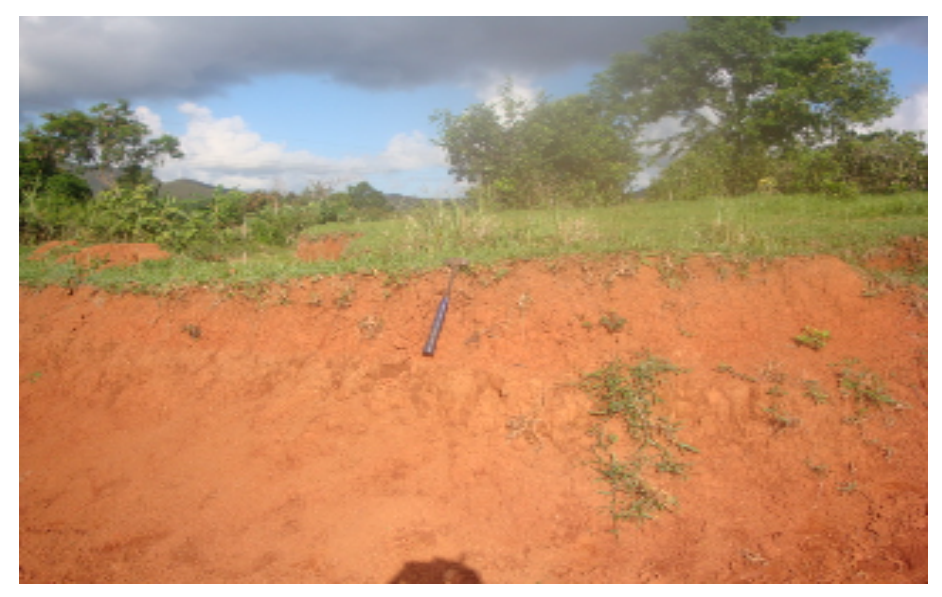

Figura 6: Corteza de meteorización.

Punto 2

Ubicación: Este punto se encuentra a $250 \mathrm{~m}$ al norte del punto anterior, $250 \mathrm{~m}$ al este del punto 4 y a $250 \mathrm{~m}$ al sur del punto 7 .

Coordenadas: X: $690282.1 \quad$ Y: 220635.7

Litologías: Se observa corteza de meteorización de color pardo, residual, no se observan grietas relícticas en superficie.

Vegetación: La vegetación es poco densa con presencia de arbustos algunos árboles de tamarindo.

Suelos: Desde el punto de vista edafológico es de color pardo - rojizo con ausencia de la capa de humus (figura 7).

Red fluvial: No existe presencia de arroyos.

Fenómenos geodinámicos: Presencia de una potente corteza de meteorización y no se observan afloramientos de rocas.

Acción antrópica: Incrementada por la acción de la construcción de la vaquería y de los asentamientos de los pobladores en la comunidad.

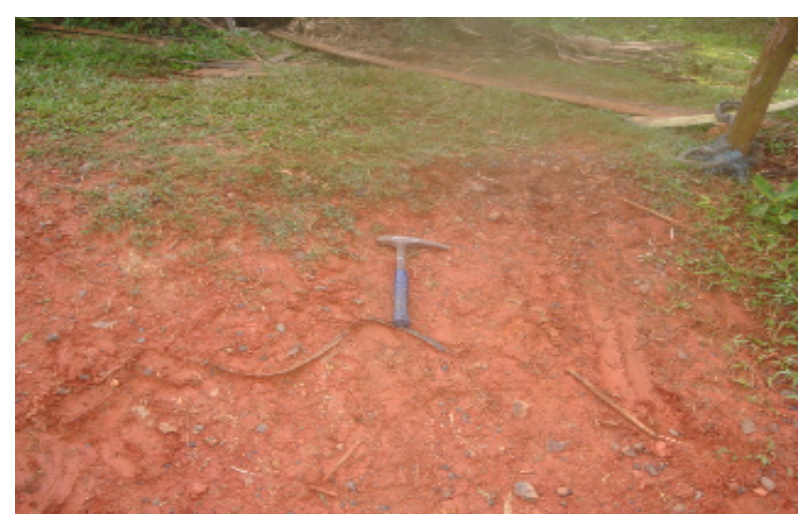

Figura 7: Suelo del área de pastoreo.

Punto 3 
Ubicación: Este punto se encuentra a $250 \mathrm{~m}$ al este del punto 1 y a $250 \mathrm{~m}$ al norte del punto 4.

Coordenadas: X: $690033.9 \quad$ Y: 220882.1

Litologías: Corteza de meteorización de color pardo-rojizo con tonalidad amarillenta, afloran las rocas ultrabásicas agrietadas, con alto grado de meteorización, fragmentadas, con desarrollo de procesos erosivos como cárcavas, se observan pequeños fragmentos de rocas con diámetro variable $\% 5$ y $15 \mathrm{~cm}$, originados por los procesos de meteorización de la roca.

Estructura: Presencia de agrietamiento con grietas pequeñas cerradas, roca serpentinítica muy meteorizada, alterada, que se rompen con facilidad.

Vegetación. La vegetación es poco densa constituida por arbustos de guayaba e icaco.

Suelos: Suelo meteorizado de color rojo oscuro (figura 8).

Red fluvial: No existe presencia de arroyos.

Fenómenos geodinámicos: Meteorización representada por una potente corteza de intemperismo. Se observan procesos erosivos representados por el desarrollo de cárcavas.

Acción antrópica: Incrementada por la acción de la construcción de la vaquería y de los asentamientos de los pobladores en la comunidad.

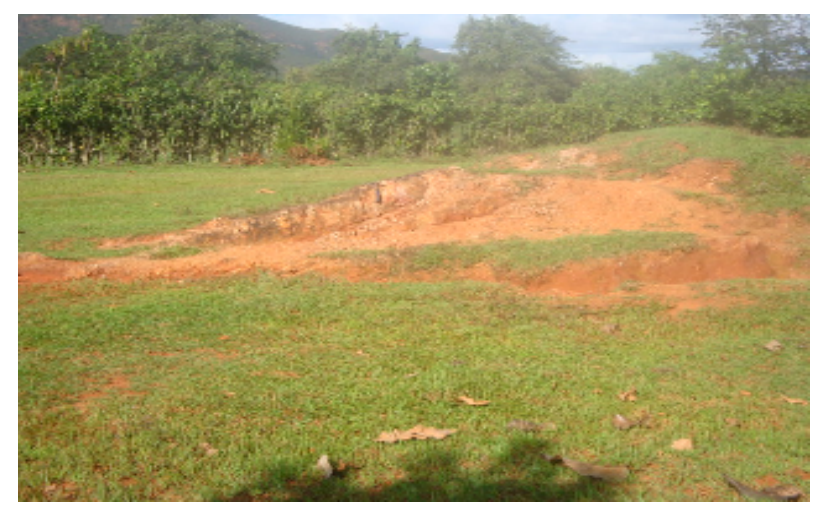

Figura 8: Afloramiento del punto 3.

Punto 4

Ubicación: Este punto se encuentra a $250 \mathrm{~m}$ al sur del punto anterior, a $250 \mathrm{~m}$ al este del punto 2, a $250 \mathrm{~m}$ al norte del punto 8 y $250 \mathrm{~m}$ al oeste del punto 5 .

Coordenadas: X: $690282.1 \quad$ Y: 220882.1

Litología: Corteza de meteorización de color pardo claro, con fragmentos de rocas Tobas con diámetro entre 1 y $2 \mathrm{~cm}$, muy alterados y meteorizados.

Estructura: Presencia de fragmentos de rocas del complejo ofiolítico muy alteradas y agrietadas, sin cúmulos básicos.

Vegetación: Poco densa con presencia de arbustos medianos como guayaba. 
Suelos: Presentan una coloración amarillenta rojiza, muy erosionados mediante pequeñas cárcavas (figura 9), pocos maduros porque se observan fragmentos de rocas muy alteradas que dificulta su caracterización, no se delimitan los horizontes del suelo, capa de humus entre 4-5 cm.

Red fluvial: No existe presencia de arroyos.

Fenómenos geodinámicos: Presencia de rocas muy alteradas, predominio de erosión, fundamentalmente acarcavamiento producto a las abundantes precipitaciones.

Acción antrópica: Incrementada por la acción de la construcción de la vaquería y de los asentamientos de los pobladores en la comunidad.

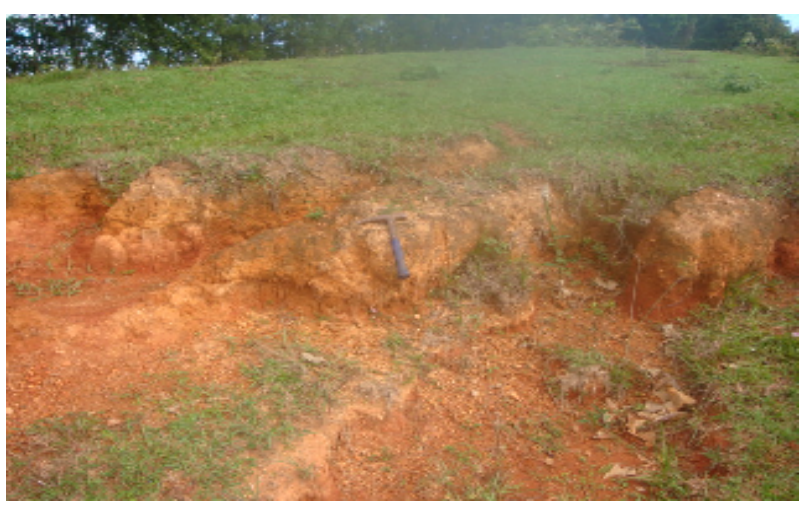

Figura 9: Erosión por cárcava.

Punto 5

Ubicación: Este punto se encuentra a $250 \mathrm{~m}$ al este del punto anterior y a $250 \mathrm{~m}$ al norte del punto 9.

Coordenadas: X: $690282.1 \quad$ Y: 221128.6

Litología: Corteza de meteorización de color rojo oscuro, se observan pequeños afloramientos de rocas ultrabásicas, específicamente peridotita serpentinizada muy alterada.

Estructura: Se encuentran fragmentos de rocas que oscilan de $5-20 \mathrm{~cm}$ que pueden haber sido transportadas de las zonas más altas.

Vegetación: La vegetación es poco densa, se observan algunos individuos de guayaba.

Suelos: Desde el punto de vista edáfico se observa una coloración rojo intensa. Incipiente capa de humus, aproximadamente $2 \mathrm{~cm}$.

Red fluvial: Se observa un arroyo intermitente que corre de este-oeste, en presencia de abundantes precipitaciones.

Fenómenos geodinámicos: Se observan procesos de meteorización y erosión mediante cárcavas (figura 10).

Acción antrópica: Incrementada por la acción de la construcción de la vaquería y de los asentamientos de los pobladores en la comunidad. 


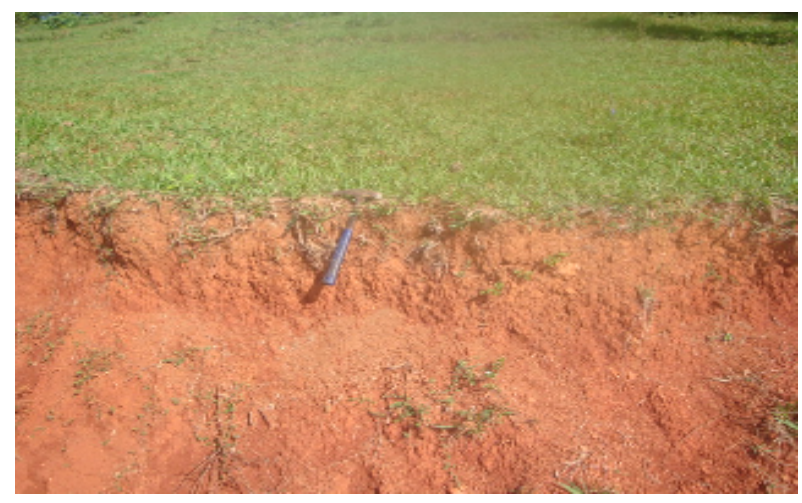

Figura 10: Erosión por cárcava.

Punto 6

Ubicación: Este punto se encuentra a $250 \mathrm{~m}$ al oeste del punto 7 y a $250 \mathrm{~m}$ al norte del punto 11 .

Coordenadas: X: $690530.4 \quad$ Y: 220389.3

Litología: Corteza de meteorización intensa, en presencia de procesos erosivos por la formación de cárcavas; se observan cúmulos máficos (gabros) porque la coloración se transforma a naranja violácea con pequeñas partes de minerales, de coloración más claras (arcilla caulinítica); afloran fragmentos de rocas alteradas y muy meteorizadas.

Estructura: Presencia de rocas meteorizadas, muy fragmentadas con diámetro de $2-8 \mathrm{~cm}$.

Vegetación: La vegetación es poco densa, en presencia de algunos arbustos, entre ellos el tamarindo.

Suelos: Desde el punto de vista edáfico se observan suelos con una coloración rojiza oscura con tonalidades violáceas que denota un componente arcilloso (caolítica), la presencia de rocas alteradas (figura 11).

Red fluvial: No existe presencia de arroyos.

Fenómenos geodinámicos: Presencia de procesos de meteorización, erosión por cárcavas y acumulación.

Acción antrópica: Incrementada por la acción de la construcción de la vaquería y de los asentamientos de los pobladores en la comunidad.

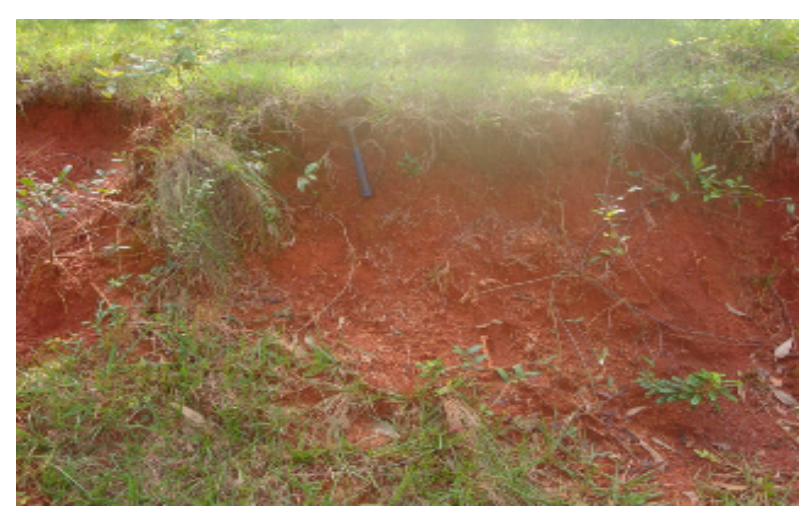

Figura 11: Suelos de pastoreo. 


\section{Punto 7}

Ubicación: Este punto se encuentra a $250 \mathrm{~m}$ al este del punto anterior, a $250 \mathrm{~m}$ al oeste del punto 8 , a $250 \mathrm{~m}$ al norte del punto 12 y a $250 \mathrm{~m}$ al sur del punto 2 .

\section{Coordenadas: X: $690530.4 \quad$ Y: 220635.7}

Litologías: Corteza de meteorización de color rojo oscuro, en presencia de afloramientos de rocas básicas agrietadas (gabros), con incremento de arcilla caolinita, con un alto grado de meteorización y fragmentación. Al lado izquierdo del punto se observan las rocas prexistentes muy alteradas, agrietadas, fragmentadas y meteorizadas.

Estructura: Presencia de agrietamiento con microgrietas cerradas, afloran fragmentos de rocas tanto de gabros como de peridotitas.

Vegetación: La vegetación es poco densa constituida por arbustos de guayaba e icaco.

Suelos: Desde el punto de vista edáfico se puede observar una coloración rojo intensa, se desarrolla según varios horizontes, roca alterada-horizonte arcilloso con minerales oxidados perdigones-capa de humus (figura 12).

Red fluvial: No existe presencia de arroyos.

Fenómenos geodinámicos: La corteza de meteorización es potente. Se observan procesos erosivos mediante cárcavas y la acumulación de sedimentos en los pies de laderas.

Acción antrópica: Incrementada por la acción de la construcción de la vaquería y de los asentamientos de los pobladores en la comunidad.

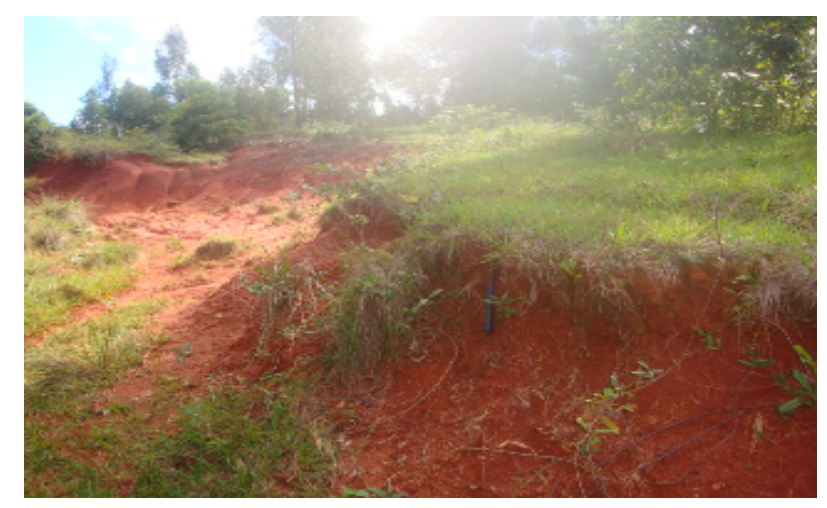

Figura 12: Suelos del área de pastoreo.

Punto 8

Ubicación: Este punto se encuentra a $250 \mathrm{~m}$ al este del punto anterior, a $250 \mathrm{~m}$ al oeste del punto 9 , a $250 \mathrm{~m}$ al norte del punto 13 y $250 \mathrm{~m}$ al sur del punto 4 .

\section{Coordenadas: X: $690530.4 \quad$ Y: 220882.1}

Litologías: Se observa corteza de meteorización de color rojo anaranjado, sin la presencia de afloramientos, pero se observan pequeños fragmentos de rocas de un diámetro aproximado de $1-2 \mathrm{~cm}$ que han sido meteorizados y transportadas desde las zonas más altas. No hay existencia de elementos de yacencia.

Vegetación: La vegetación es poco densa constituida por arbustos de guayaba y tamarindo. 
Suelos: Desde el punto de vista edáfico se observa una coloración roja intensa, con abundantes perdigones y una capa de humus incipiente, aproximadamente $2 \mathrm{~mm}$ (figura 13).

Red fluvial: No existe presencia de arroyos.

Fenómenos geodinámicos: Potente corteza de meteorización. En presencia de procesos erosivos principalmente por cárcavas.

Acción antrópica: Incrementada por la acción de la construcción de la vaquería y de los asentamientos de los pobladores en la comunidad.

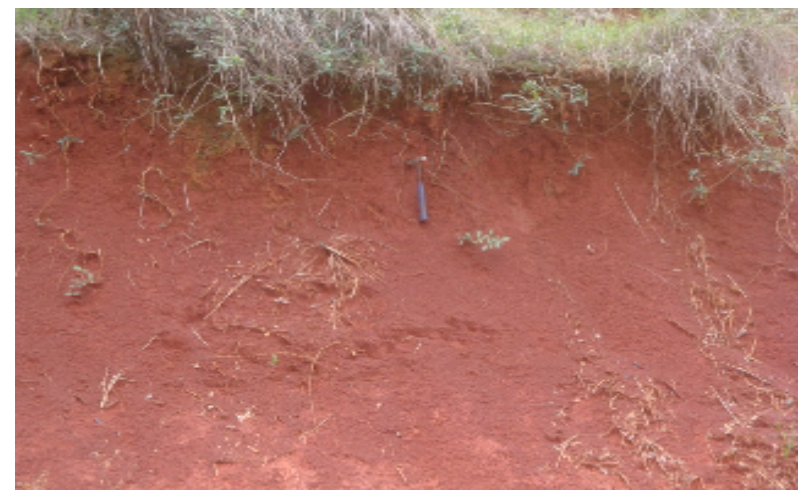

Figura 13: Suelos del área de pastoreo.

Punto: 9

Ubicación: Este punto se encuentra a $250 \mathrm{~m}$ al este del punto anterior, a $250 \mathrm{~m}$ al oeste del punto 10 , a $250 \mathrm{~m}$ al norte del punto 14 y a $250 \mathrm{~m}$ al sur del punto 5 .

Coordenadas: X: $690530.4 \quad$ Y: 221128.6

Litologías: En presencia de una corteza de meteorización de color rojo - amarillento claro, se observan fragmentos de las rocas prexistentes con predominio de un diámetro de $2-5 \mathrm{~cm}$ y algunas de $10-40 \mathrm{~cm}$, el tipo de rocas existente en este punto es Tobas y peridotitas serpentinizadas.

Estructura: No poseen agrietamiento ni elementos de yacencia.

Vegetación: La vegetación es poco densa constituida por arbustos de charrascal, helechos e icacos.

Suelos: Desde el punto de vista edáfico se observa un color rojizo claro con tonalidad naranja, capa de humus incipiente $1-2 \mathrm{~cm}$, fragmentos de rocas alteradas del suelo, lo que demuestra un suelo poco maduro (figura 14).

Red fluvial: No existe presencia de arroyos.

Fenómenos geodinámicos: Se observan fuertes procesos de meteorización y erosión por cárcavas.

Acción antrópica: Incrementada por la acción de la construcción de la vaquería y de los asentamientos de los pobladores en la comunidad. 


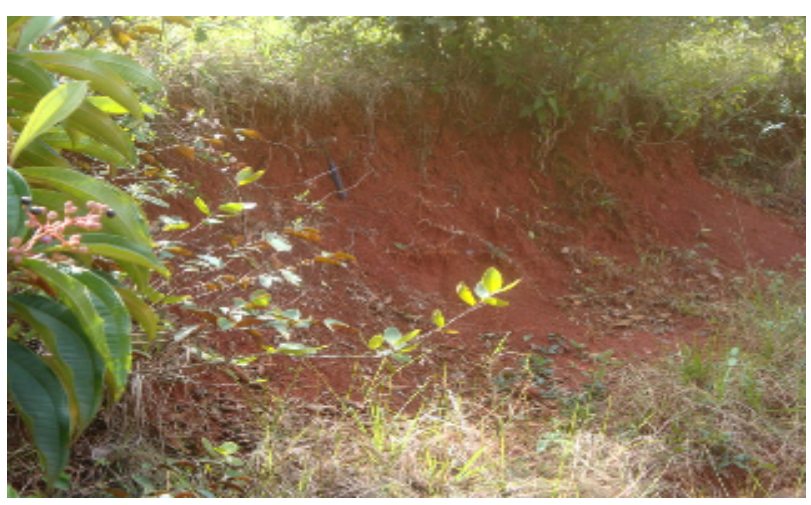

Figura 14. Suelos del área de pastoreo.

Punto: 10

Ubicación: Este punto se encuentra a $250 \mathrm{~m}$ al este del punto anterior.

Coordenadas: X: $690530.4 \quad$ Y: 221375

Litologías: Corteza de meteorización de color rojo oscuro, se observan afloramientos con una longitud de $15 \mathrm{~m}$ de rocas Tobas, además la existencia de procesos erosivos por cárcavas.

Estructura: En presencia de estratificación de las rocas, no se observan elementos de yacencia.

Vegetación: La vegetación es poco densa constituida por arbustos de guayaba e icacos.

Suelos: Desde el punto de vista edáfico se observa una coloración naranja claro, poco potente, con incipiente desarrollo de horizonte de humus (figura 15).

Red fluvial: No existe presencia de arroyos.

Fenómenos geodinámicos: Presencia de una potente corteza de meteorización y erosión por cárcavas.

Acción antrópica: Incrementada por la acción de la construcción de la vaquería y de los asentamientos de los pobladores en la comunidad.

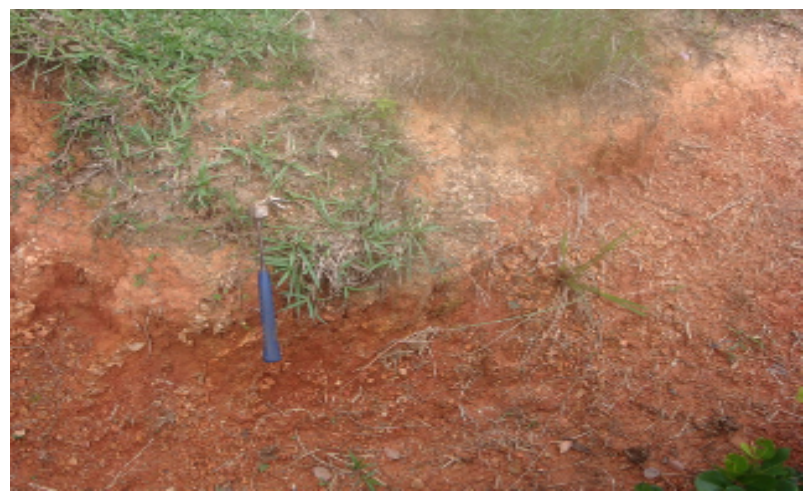

Figura 15: Suelos del área de pastoreo.

Punto 11

Ubicación: Este punto se encuentra a $250 \mathrm{~m}$ al sur del punto 6 y a $250 \mathrm{~m}$ al oeste del punto 12. 


\section{Coordenadas: X: $690778.6 \quad$ Y: 220389.3}

Litologías: Corteza de meteorización de color pardo oscuro, en presencia de afloramientos de rocas ultrabásicas agrietadas, con alto grado de meteorización y fragmentación (peridotitas serpentinizadas) (figura 16).

Estructura: Existe presencia de agrietamiento con microgrietas cerradas.

Vegetación: La vegetación es poco densa constituida por arbustos de guayaba, icaco y tamarindo.

Suelos: Desde el punto de vista edáfico se observa una coloración parda bien intensa, capa de humus incipiente.

Red fluvial: No existe presencia de arroyos.

Fenómenos geodinámicos: Presencia de una potente corteza de meteorización y procesos erosivos por cárcavas.

Acción antrópica: Incrementada por la acción de la construcción de la vaquería y de los asentamientos de los pobladores en la comunidad.

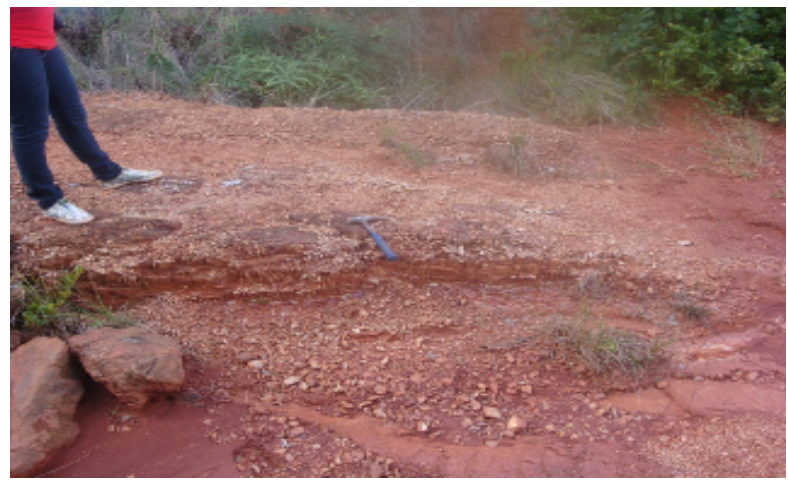

Figura 16: Afloramiento del punto 11.

Punto 12

Ubicación: Este punto se encuentra a $250 \mathrm{~m}$ al este del punto anterior, a $250 \mathrm{~m}$ al sur del punto 7 y a $250 \mathrm{~m}$ al oeste del punto 13.

Coordenadas: X: $690778.6 \quad$ Y: 220635.7

Litologías: Corteza de meteorización de color pardo claro, en presencia de afloramientos de rocas ultrabásicas agrietadas, con alto grado de meteorización y fragmentación, alrededor del punto se observan las rocas prexistentes muy alteradas, agrietadas, fragmentadas y meteorizadas. Rocas ultrabásicas serpentinizadas, no existe presencia de microgrietas.

Vegetación: La vegetación es poco densa constituida por arbustos de guayaba e icaco.

Suelos: Desde el punto de vista edáfico se observa una coloración rojo amarillenta claro, horizonte de humus incipiente (figura 17).

Red fluvial: No existe presencia de arroyos.

Fenómenos geodinámicos: Presencia de una potente corteza de meteorización, se observan procesos erosivos por cárcavas. 
Acción antrópica: Incrementada por la acción de la construcción de la vaquería y de los asentamientos de los pobladores en la comunidad.

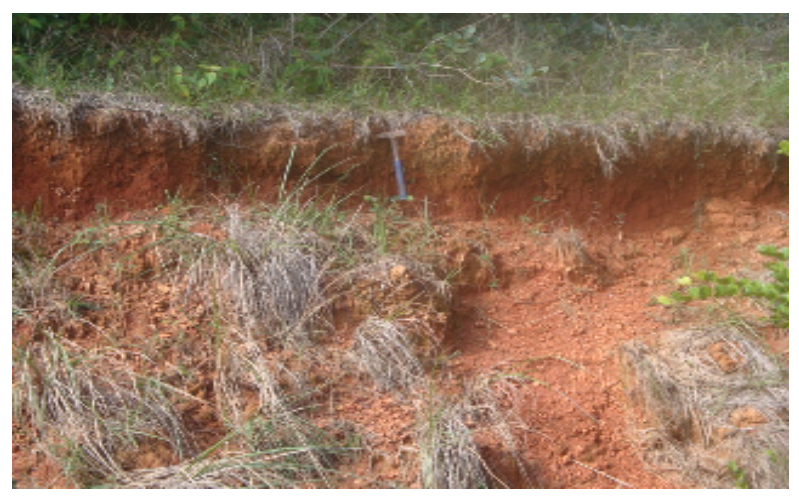

Figura 17: Suelos del área de pastoreo.

Punto 13

Ubicación: Este punto se encuentra a $250 \mathrm{~m}$ al este del punto anterior, a $250 \mathrm{~m}$ al oeste del punto 14 y a $250 \mathrm{~m}$ al sur del punto 8 .

Coordenadas: X: $690778.6 \quad$ Y: 220882.1

Litologías: Las rocas que afloran están in situ y son rocas del complejo ultramáficoserpentinizado. Se encuentran muy alteradas y agrietadas, alrededor del punto se observan las rocas prexistentes muy alteradas, agrietadas, fragmentadas y meteorizadas.

Estructura: Existe presencia de agrietamiento con microgrietas cerradas.

Vegetación: La vegetación es poco densa constituida por arbustos de guayaba e icaco.

Suelos: Desde el punto de vista edáfico se observa una coloraciónrojo intensa, con incipiente capa de humus (figura 18).

Red fluvial: No existe presencia de arroyos.

Fenómenos geodinâmicos: Presencia de una potente corteza de meteorización, se observan procesos erosivos por cárcavas.

Acción antrópica: Incrementada por la acción de la construcción de la vaquería y de los asentamientos de los pobladores en la comunidad.

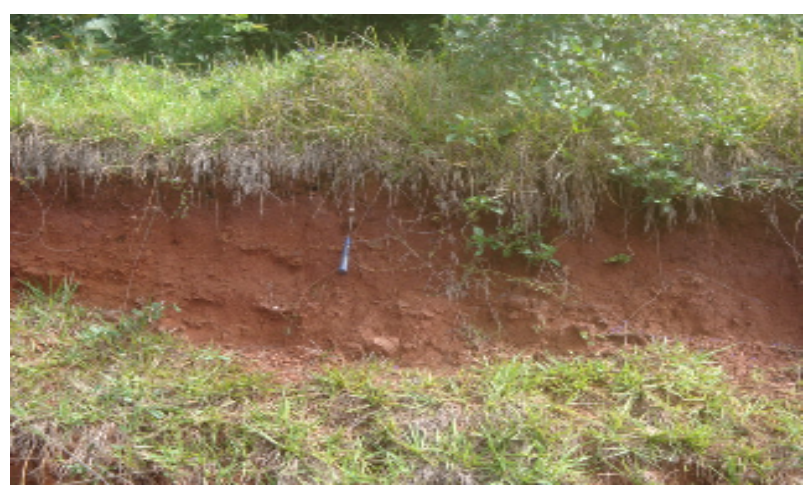

Figura 18: Suelos del área de pastoreo.

Punto 14 
Ubicación: Este punto se encuentra a $250 \mathrm{~m}$ al este del punto anterior y a $250 \mathrm{~m}$ al sur del punto 9.

Coordenadas: X: $690778.6 \quad$ Y: 221128.6

Litología: Corteza de meteorización de color pardo claro.En este punto no afloran rocas en superficie, se observan rocas deslizadas de las zonas más altas.

Vegetación: La vegetación es poco densa constituida por arbustos de guayaba e icaco.

Suelos: Desde el punto de vista edáfico se observa una coloración rojiza con tonalidad clara. No se pueden discriminar los diferentes horizontes del suelo (figura 19).

Red fluvial: No existe presencia de arroyos.

Fenómenos geodinámicos: Presencia de una potente corteza de meteorización y erosión por cárcavas.

Acción antrópica: Incrementada por la acción de la construcción de la vaquería y de los asentamientos de los pobladores en la comunidad.

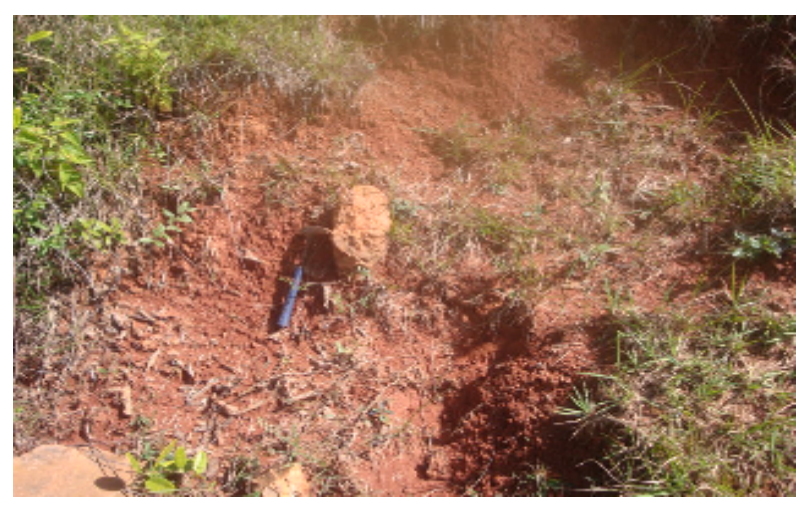

Figura 19: Suelos del área de pastoreo.

\section{DISCUSIÓN DE LOS RESULTADOS}

Los trabajos de campo desarrollados en el área de pastoreo permiten determinar las características geológicas y ambientales, entre las cuales es de destacar el predominio del suelo cubriendo toda la superficie y solo en dos puntos, 3 y 11, pudieron observarse la roca madre con un alto grado de meteorización y fragmentación.

En el área de pastoreo se evidenció la ocurrencia de procesos geodinámicos de superficie como la meteorización, la erosión y la acumulación, esta última referida a los pies de laderas.

La meteorización, fenómeno físico - geológico más importante en el área de estudio, responsable de la formación de potentes cortezas lateríticas sobre rocas básicas y ultrabásicas, se pone de manifiesto a través de un suelo de color rojo-anaranjado, sobre el cual se ha desarrollado una incipiente capa de humus.

En toda el área hay un predominio de los procesos de erosión superficial, puesta de manifiesto fundamentalmente en la erosión por cárcavas, condicionada por tres factores fundamentales: 
La acción de las precipitaciones.

La inclinación del terreno que influye en la pérdida de una parte del perfil de suelo.

La respuesta erosiva de los suelos en función del mal manejo y uso.

Este proceso se hace más intenso por la propia actividad de pastoreo que sobre el área se realiza, que elimina la cobertura vegetal, acompañado de las malas prácticas agrotécnicas, al no aplicarse planes de mejoramiento y rehabilitación de los sectores afectados.

A pesar del predominio de los procesos de erosión, en algunos puntos (5 y 6) pudo observarse la acumulación de sedimentos, materiales alterados y transportados previamente y que han quedado temporalmente al pie de las pequeñas laderas. Sin la presencia de vegetación.

\section{CONCLUSIONES}

La presente investigación con el cumplimiento de los objetivos propuestos nos permite concluir que:

Al realizarse el análisis de las características geológicas y edafológicas predominantes en el área de pastoreo, se observó la incidencia de los procesos de erosión y meteorización que condicionan las características del suelo. Se evidencia el pobre desarrollo de la vegetación y la mala conservación de los recursos edáficos y la necesidad y posibilidad de realizar la división del área por cuartones para que potencie el mejor uso y la mayor eficiencia productiva.

\section{REFERENCIAS BIBLIOGRÁFICAS}

Batista, J. (1998). Caracterización geológica y estructural de la región de Moa a partir de la interpretación del levantamiento aeromagnético 1: 50 000. Tesis de maestría. Instituto Superior Minero Metalúrgico de Moa, Departamento de Geología.

Carreño-Vega. B; González-Febles M.J (2005) Universidad Agraria de La Habana. La investigación de suelos erosionados: Métodos e Índices de diagnóstico. ISSN 02585979 Minería y Geología v. 21 n. 2, 2005.

CCD/PNUMA, (1995). Convención de las Naciones Unidas de Lucha contra la Desertificación en los países afectados por sequía grave o desertificación, en particular en África. Texto con anexos. Suiza, 71p.

Chaviano, A. (2016). Algunas consideraciones de rehabilitación minera en la minería del Níquel: Municipio de Moa, Cuba. DELOS: Desarrollo Local Sostenible, 4: 10.

García, D. (2018). Tesis de diploma. Análisis del proceso de degradación de los suelos en la cuenca del río Cayo Guam. Instituto Superior Minero Metalúrgico de Moa. Departamento de Geología.

Fernández, Z. (2017). Plan de MIZC para minimizar el azolvamiento en la bahía de Cayo Moa. Tesis de maestría. Universidad de Oriente. 
Martínez. B; et al. (2014), en su artículo "Desarrollo de una metodología de detección de cambios en zonas susceptibles de procesos de degradación". Dept. de Termodinámica Fac. De Física, Universidad de Valencia. Teledetección, Medio Ambiente y Cambio Global (2010) 110-113.

Ramírez, Y. (2015). Mapa geotécnico del área de emplazamiento de la Planta Ferroníquel Minera S.A. Tesis de grado. Instituto Superior Minero Metalúrgico de Moa, Departamento de Geología.

Rodríguez, A. (1999). Estudio morfotectónico de Moa y áreas adyacentes para la evaluación de riesgos de génesis tectónicas. Tesis doctoral. Instituto Superior Minero Metalúrgico de Moa, Departamento de Geología.

Rodríguez, Y. (2015). Factores que influyen en la desertificación en el sector Cerro-MirafloresPlaya La Vaca, municipio Moa. Tesis de diploma. Instituto Superior Minero Metalúrgico de Moa. Departamento de Geología.

Yakabi, k. (2016). Estudio de las propiedades edáficas que determinan la fertilidad del suelo en el sistema de andenería de la comunidad campesina, San Pedro de Laraos, provincia de Huarochirí, Lima. Tesis de grado. Universidad Católica del Perú, Departamento Geografía y Medio ambiente. 